\title{
Kinematics Analysis of a Novel Five-Degree-of-Freedom Spatial Parallel Micromanipulator
}

\author{
Daniel Prusak, Konrad Kobus, and Grzegorz Karpiel \\ Department of Robotics and Mechatronics, Faculty of Mechanical Engineering and Robotics, \\ AGH University of Science and Technology, Al. Mickiewicza 30, 30-059 Krakow, Poland \\ Correspondence should be addressed to Daniel Prusak; daniel.prusak@agh.edu.pl
}

Received 4 June 2013; Revised 7 November 2013; Accepted 11 November 2013; Published 25 March 2014

Academic Editor: Yangmin Li

Copyright (C) 2014 Daniel Prusak et al. This is an open access article distributed under the Creative Commons Attribution License, which permits unrestricted use, distribution, and reproduction in any medium, provided the original work is properly cited.

A study of the inverse kinematics for a five-degree-of-freedom (DOF) spatial parallel micromanipulator is presented here below. The objective of this paper is the introduction of a structural and geometrical model of a novel five-degree-of-freedom spatial parallel micromanipulator, analysis of the effective and useful workspace of the micromechanism, presentation of the obtained analytical solutions of the microrobot's inverse kinematics tasks, and verification of its correctness using selected computer programs and computation environments. The mathematical model presented in this paper describes the behaviour of individual elements for the applied 2-DOF novel piezoelectric actuator, resulting from the position and orientation of the microrobot's moving platform.

\section{Introduction}

Over the past years much attention has been devoted to the analysis of parallel micromanipulators $[1,2]$. These mechanisms have a major advantage over serial microrobots, reflected inter alia in their accuracy, repeatability, and speed, with which the desired position can be achieved [3-5]. On the other hand, the parallel manipulators have a relatively smaller workspace, limited by the maximum arms lengths, angle values at the joints, and their dimensions.

Moreover, the main difficulty with parallel manipulators (both in micro- and macroscale) is the complexity of controlling their movement. Kinematic structures of various types of parallel mechanisms may contain serial, parallel, or serial-parallel (hybrid) types of linkages, mounted to the platforms by universal joints [6-10]. The desired position and orientation of the moving platform are achieved by combining the linear and angular lengths of the robot's arms, transformed by their degrees of freedom into three positional and orientational ones for the operating member [11-13]. The problem of inverse kinematics task for spatial parallel manipulators can be defined as finding the linkage's lengths needed to position the moving platform along a specified geometric path, aligned accordingly with the desired orientation [14-17]. The solution to this problem can sometimes be indeed very complex and less suitable for realtime computing. However, the computation of length for each arm can be carried out independently, which can additionally speed up the process [18]. This procedure is used to guide the moving platform in controlling its movement $[16,19]$. Quoted features constitute the greatest importance when it comes to designing and constructing parallel platforms, operating in micro- or nanometric scales [20-23]. Thus far, several attempts have been made to adapt the parallel mechanisms to build a spatial parallel micropositioning platforms, with various effects, shown in $[4,10,24-33]$.

Another problem is the precision of a micromanipulator, which is determined not only by the deformations of the structure, caused by external forces, but also by the strain derived from changes in ambient temperature [34]. To compensate those deformations, occurring under certain conditions of environment, the proposed project solution is to equip a standard spatial three-axes parallel microrobot (tripod) with additional two rotational degrees of freedom, which - through the control system-will result in decreasing inaccuracies resulting from thermal expansion of the micromechanism's components [35]. Additional degrees of freedom, in comparison to previous versions of micromanipulators, constructed in the Department of Robotics and Mechatronics at the AGH University of Science 


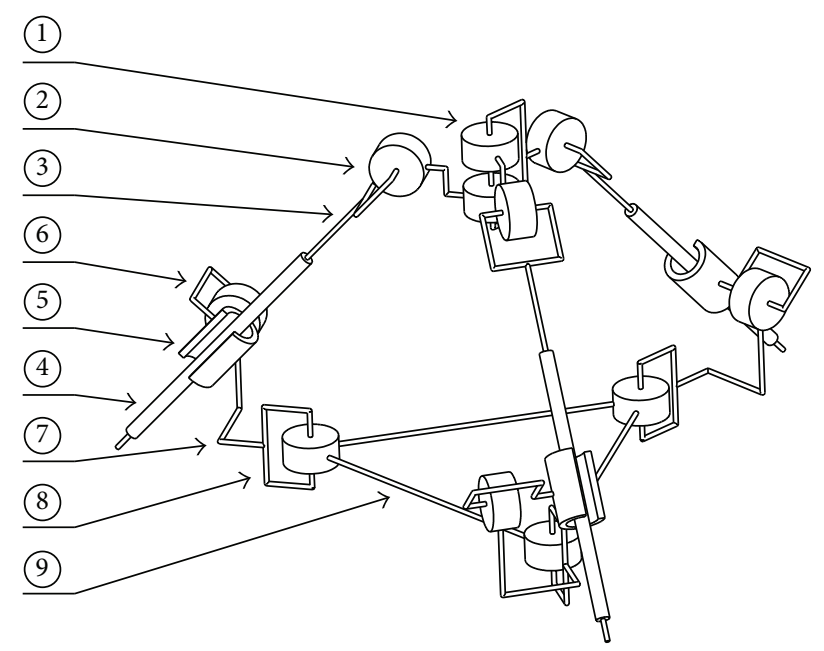

FIGURE 1: Structure of a 5-DOF spatial parallel micromanipulator: (1): moving platform, (2), (6), and (8): revolute joint, (3): connector, (4): actuator shaft, (5): stator, (7): support, and (9): microrobot's base.

and Technology, were obtained by introducing a rotary movement to the microrobot's arms [36]. It has been achieved by using completely new, specifically design movement transmission system based on three novel type piezoelectric actuators with two degrees of freedom, generating both linear and rotational movement $[37,38]$. The movement control of the spatial parallel micromanipulator is restricted mainly to inverse kinematics, resulting from the complexity of the forward kinematics relations.

\section{Geometric Structure of 5-DOF Parallel Micromanipulator}

The structure of the proposed 5-degree-of-freedom spatial parallel micromanipulator is shown in Figure 1. The presented mechanism consists of a fixed platform, hereinafter referred to as the base (9, and has three piezoelectric actuators with two degrees of freedom. Each actuator combining linear and rotational movement, acting like a pair of prismatic $(P)$ and revolute $(R)$ joints, can generate independent linear $\mathbf{l}_{\mathbf{i}}$ and angular $\beta_{i}$ displacements along its main (symmetry) axis. The orientation of the base plain $x-y$ will be referred to as horizontal. The actuators are placed on passive (nonactuated) rotary $(\mathrm{R})$ joints $(6$, whose axis of rotation is perpendicular to the actuator symmetry axis and parallel to the horizontal base plane. They link them with the base and will be hereinafter referred to as the supports 7 , which are placed on the base, on $\mathrm{R}$ radius circle and relative angles, $90^{\circ}, 210^{\circ}$, and $360^{\circ}$, respectively, to the $x$-axis (between the first and the following drive). The supports are placed on the base by passive rotary (R) joints (8), whose axis of rotation is perpendicular to the basis $x-y$ plane. The stator (5) of the used piezoactuators is mounted on bearings in the supports by means of specially mounted rotary $(\mathrm{R})$ nonactuated clamps. The shafts of all the drives (4) are attached to the moving platform unit (1) using properly designed passive revolute $(R)$ joints (3), equipped with rotary bearings (2) with rotary axis always parallel to the moving platform (1). The moving platform (1) consists of three

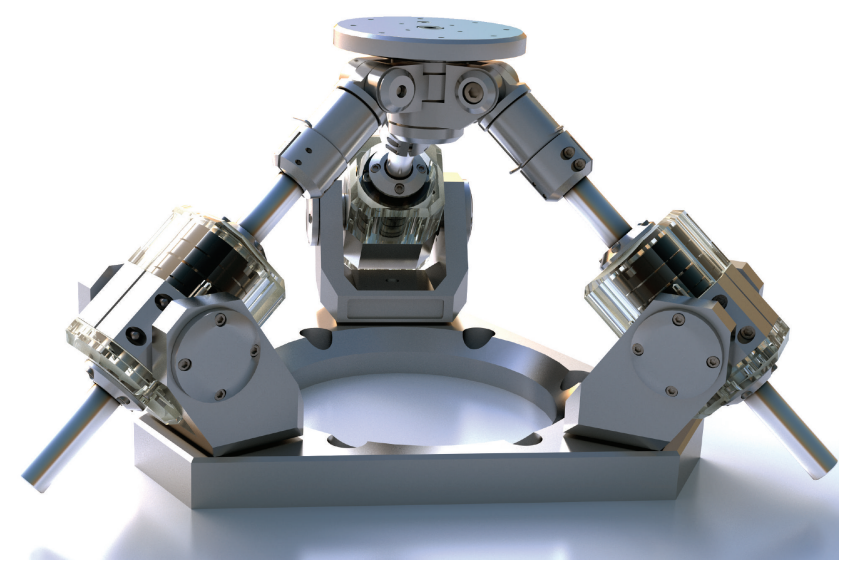

FIGURE 2: Novel 5-DOF spatial parallel micromanipulator.

specially designed revolute joints $(R)$ which has one common axis of rotation coinciding with the main axis of the platform. Thus, the base is attached to the moving platform by three identical $\mathrm{R}-\mathrm{R}-(P-R)-\mathrm{R}-\mathrm{R}$ (or in other notation $\mathrm{R}-\mathrm{R}-\mathrm{C}-\mathrm{R}-\mathrm{R}$ ) linkages, as shown in Figure 1.

The most important feature of the presented micromechanism is the implementation of additional rotational $(R)$ movement of the arms around their own axis of symmetry, in addition, giving the spatial parallel micromanipulator up to five degrees of freedom (three translational and two angular movements of moving platform). Moreover, an asset of the considered structure is that the actuators are mounted very closely to the base, without any offsets relative to the moving platform. It simplifies the geometry and the mechanical design, reduces the overall inertia of the mechanism, and increases its mobility payload capabilities, which is an especially important feature in many micro-/nanoapplications required to avoid inducing vibrations of the structure. The view of the 5-DOF spatial parallel micromanipulator is shown in Figure 2.

The constructions overall complexity arrangements have a great impact on their development process, Błąd: Nie znaleziono źródła odwołania. The connections between working elements must be specially designed, most often due to particular conditions in which the drives selected for the manipulator work, but also depending on mathematical models describing geometric, kinematic, and dynamic parameters, characterized by a high degree of complexity. It is usually in opposition to the sophistication level of the mechanical solutions. The simpler the mechanical design is, the more difficult the mathematical model becomes. Vice versa the more the transparent computational model is being used, the more challenging the mechanical design is. In some cases, hardly obtained mathematical model is so difficult to implement that it can complicate the designing and building process of the control system. It may be impossible to control a spatial parallel microrobot in real time. On the other hand, adjustments aimed at possible simplifications in the calculation model can lead to a significant deterioration of important parameters, characterizing micromanipulators. Interdisciplinary nature, involved when 


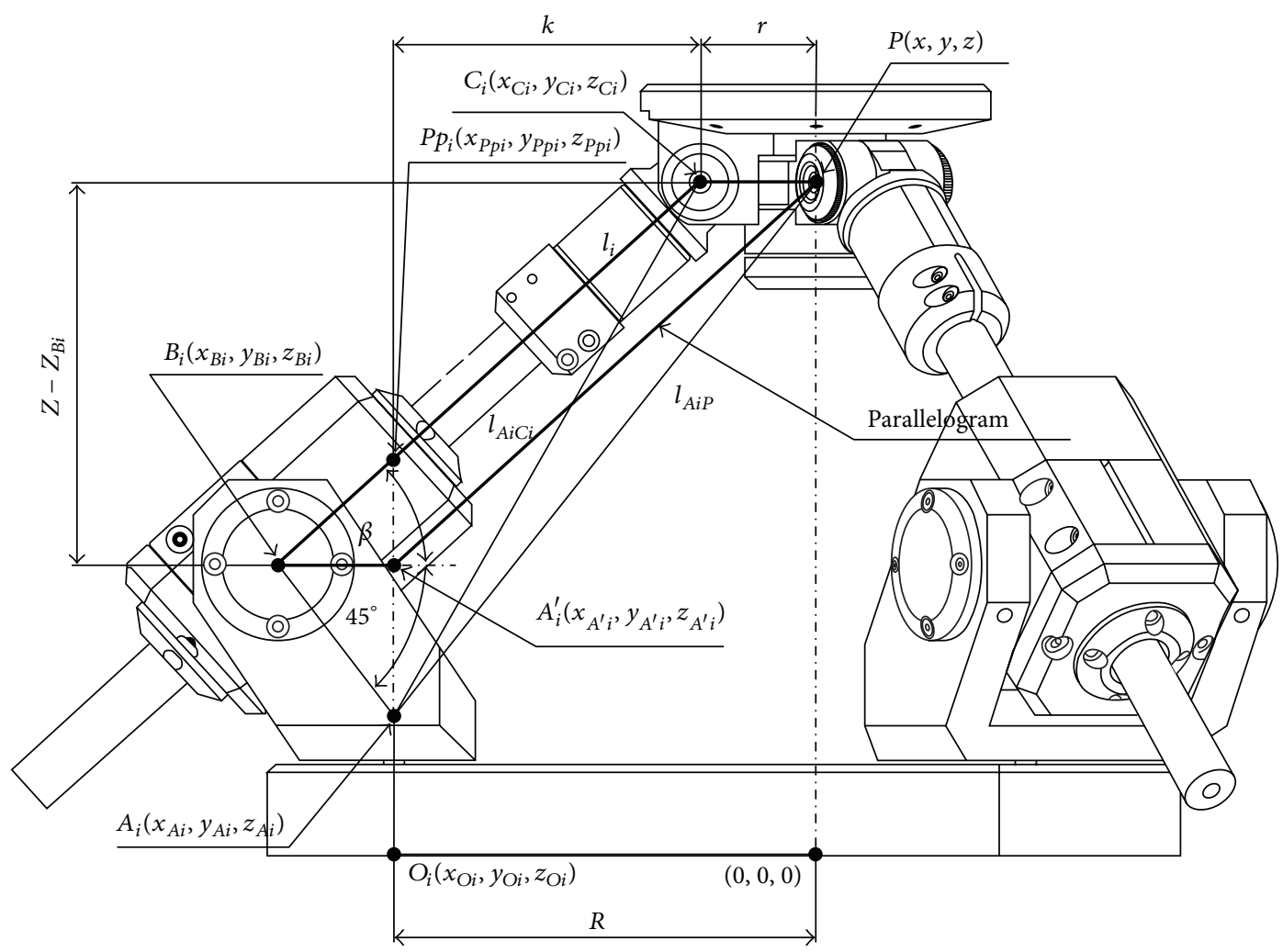

FIgURE 3: Geometry characteristic of the microrobot's $i$ th arm.

designing microrobots, makes it necessary to deal with problems of computing, simulation, control, and numerous physical phenomena.

\section{The Inverse Kinematics Problem}

The inverse kinematics determines the displacements of the linkages (arms) with respect to the known position and orientation of the end effector of the spatial parallel micromanipulator [14-17]. By using a linear-rotary piezoelectric drives, it is possible to independently control the orientation of the moving platform and position in the Cartesian system. The design of the micromechanism presented in this paper has a unique geometry [9]. Points that are being determined through the axes of the rotating joints of individual linkages form a closed parallelogram, as shown in Figure 3 [6]. This means that, at any position, the moving platform linking together all the arms of the microrobot can be treated as a spherical joint [7]. Considering technical aspects of presented construction, it should be noted that this situation exists only if no rotational movement is made, which means only when the main axis of moving platform is perpendicular to the base. The length of the respective sides of thus formed parallelogram determines directly the distance measure from the base of the micromanipulator to the moving platform [8]. This is a valuable feature, which is essential in determining the inverse kinematics of the presented 5-DOF spatial parallel microrobot.

First step in geometric model calculations is to determine proper vector representation. The coordinates axes of the micromanipulator's base (the inertial frame) are denoted by $O X Y Z$, while those of the moving platform are denoted by $P^{\prime} X^{\prime} Y^{\prime} Z^{\prime}$, as shown in Figures 3 and 4 . To simplify the presented kinematic model, the $y$-axis of the inertial frame is aligned with one of the piezoelectric actuators supports, linking them with the base structure in points marked as $\mathbf{A}_{\mathbf{i}}$ by means of a passive revolute joint, being the first of the R$\mathrm{R}-(P-R)-\mathrm{R}-\mathrm{R}$ linkage. The ends of the individual supports are pointing towards the second passive revolute joint of the R-R$(P-R)-\mathrm{R}-\mathrm{R}$ linkage, of which rotational axis positions will be referred to as $\mathbf{B}_{\mathbf{i}}$. The $X$ - and $Y$-axis lie in the plane of the fixed platform (the horizontal plane of the base), while the $z$-axis is defined as normal to this plane (the vertical plane of the base), pointing upwards, thereby forming the right-handed orthogonal frame. The origin of this frame is located at the circle center $O\left(x_{o}, y_{o}, z_{o}\right)$ of the base. The $X^{\prime} Y^{\prime}$-plane of the mobile frame is aligned and fixed with the moving platform, with the origin $P\left(x_{p}, y_{p}, z_{p}\right)$ at its centre, through which the rotational axis of the individual revolute joints of the platform coincides with each other. The $Y^{\prime}$-axis points towards one of the passive revolute attachment joints, located in point $\mathbf{C}_{\mathbf{i}}$. The $Z^{\prime}$-axis completes the right-handed coordinate system and points upward when the upper platform is in its initial position.

According to designations presented in Figures 3, 4, 5, 6, and 7 , the following vectors and geometric parameters are determined:

(i) $\mathbf{p}=\left[x_{p}, y_{p}, z_{p}\right]^{T}$ is the vector from point $O(0,0,0)$, the origin of the fixed platform (the base 


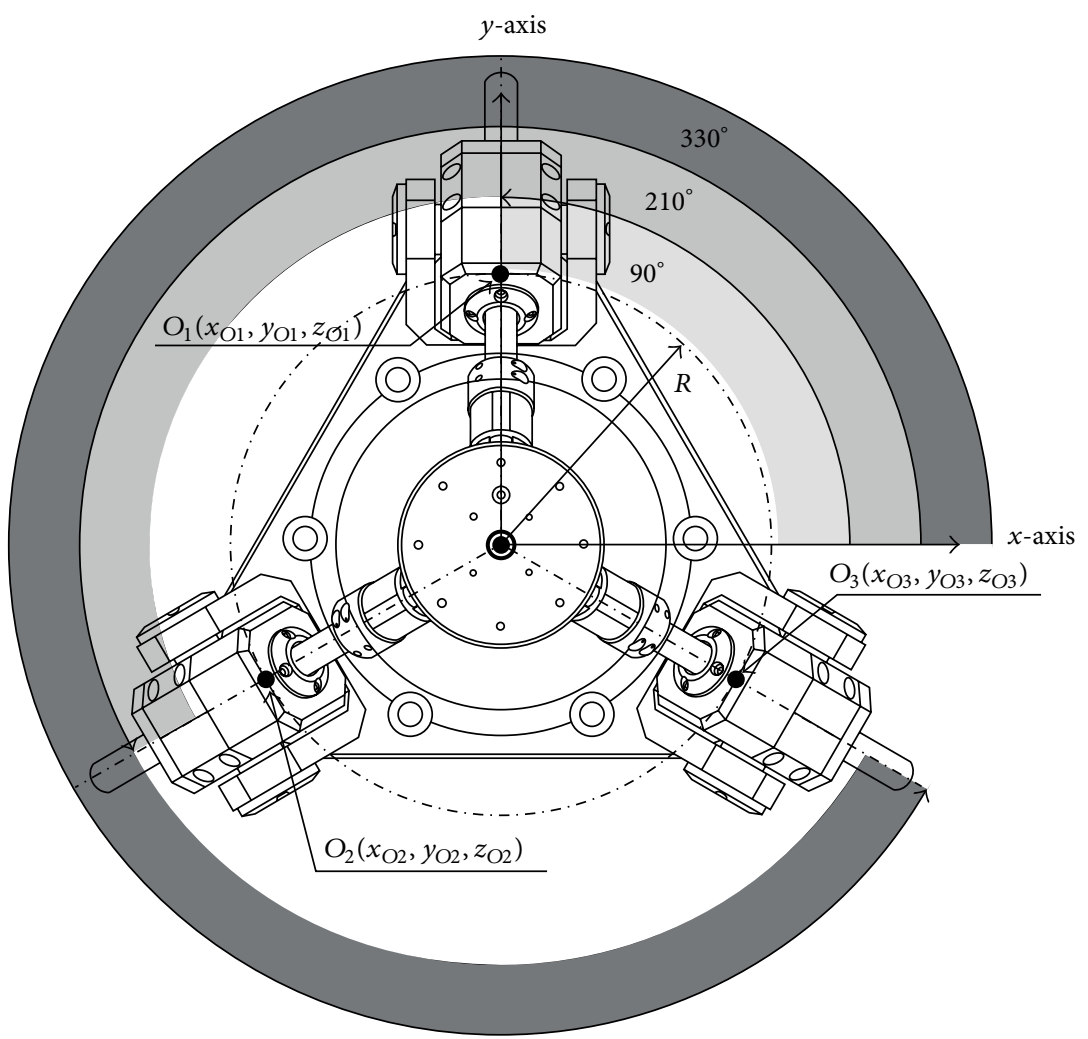

FIGURE 4: Geometry characteristic of the microrobot's base.

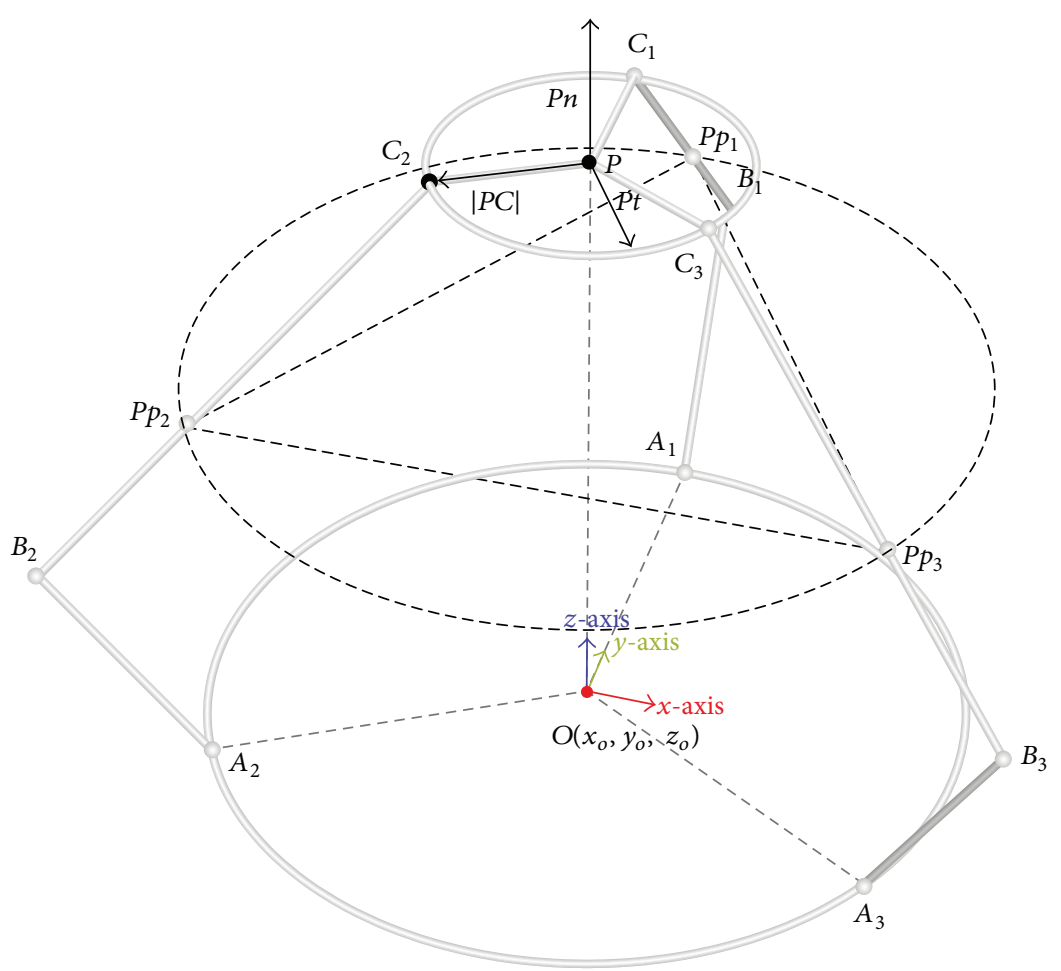

FIGURE 5: Cabri 3D geometrical model of micromanipulator with vector $P C$ connected in point $P$ with the moving platform. 


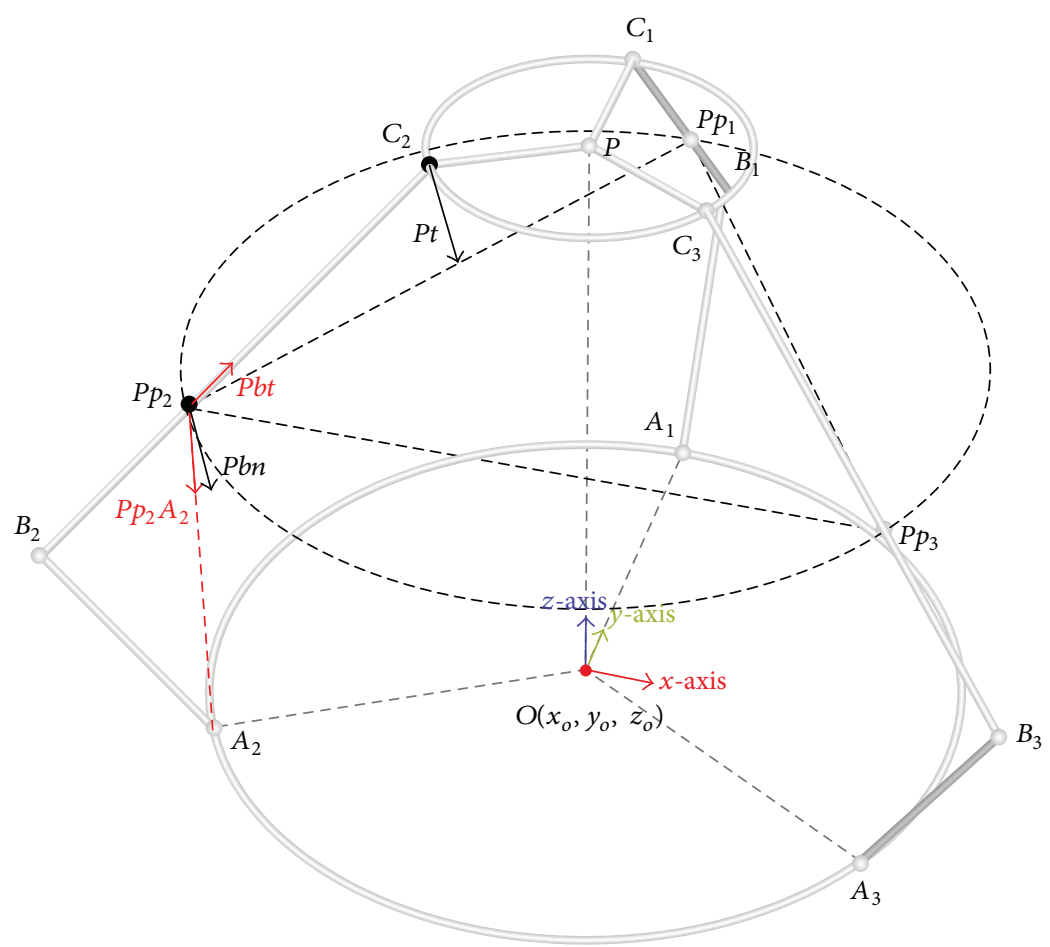

FIGURE 6: Cabri 3D geometric model of micromanipulator with vectors indicates angular translation $\delta$.

of micromanipulator), to point $P=\left(x_{p}, y_{p}, z_{p}\right)$, the origin of the moving platform;

(ii) $\mathbf{p}^{\mathbf{n}}=\left[p_{x}^{n}, p_{y}^{n}, p_{z}^{n}\right]$ and $\mathbf{p}^{\tau}=\left[p_{x}^{\tau}, p_{y}^{\tau}, p_{z}^{\tau}\right]$ represent vectors normal and tangent to the moving platform;

(iii) $\mathrm{R}$ is the radius of the circle on which the supports of the piezoelectric actuators are placed (position of the first revolute joints of the $i$ th linkage);

(iv) $\mathbf{O}_{\mathbf{i}} \mathbf{A}_{\mathbf{i}}=\left[O_{i} A_{i x}, O_{i} A_{i y}, O_{i} A_{i z}\right]^{T}$ is the vector from the support attachment point $O_{i}$ to the construction point $A_{i}$ of the $i$ th linkage; it should be noted that $\left|\mathbf{O}_{\mathbf{i}} \mathbf{A}_{\mathbf{i}}\right|=$ $O_{i} A_{i}$ is the constant distance for each arm;

(v) $\mathbf{A}_{\mathbf{i}} \mathbf{B}_{\mathbf{i}}=\left[A_{i} B_{i x}, A_{i} B_{i y}, A_{i} B_{i z}\right]^{T}$ is the vector from point $A_{i}$ to point $B_{i}$, through which the axis of the second revolute joint of the $i$ th linkage goes; it should be noted that $\left|\mathbf{A}_{\mathbf{i}} \mathbf{B}_{\mathbf{i}}\right|=A_{i} B_{i}$ is the constant distance for each arm;

(vi) $\mathbf{l}_{\mathbf{i}}=\left[l_{i x}, l_{i y}, l_{i z}\right]^{T}$ is the vector from point $B_{i}$ of the $i$ th lower attachment point of the actuator's stator (revolute joint) to point $C_{i}$ of the $i$ th upper attachment point of its shaft (revolute joint); it should be noted that $\left|\mathbf{I}_{\mathbf{i}}\right|=l_{i}$ represents various displacement of the $i$ th 2-DOF piezoelectric actuator;

(vii) $\mathbf{P}_{\mathbf{i}} \mathbf{C}_{\mathbf{i}}=\left[P_{i} C_{i x}, P_{i} C_{i y}, P_{i} C_{i z}\right]^{T}$ is the vector from point $C_{i}$ of the $i$ th upper attachment point of the actuator's shaft (revolute joint) to the origin of the moving platform $P_{i}$; it should be noticed that $\left|\mathbf{P}_{\mathbf{i}} \mathbf{C}_{\mathbf{i}}\right|=r$ is the constant distance for each arm; (viii) $k$ is an auxiliary length, defined in $X Y$-plane, between point $O_{i}$ of the $i$ th support position on the base and point $C_{i}$ of the $i$ th upper attachment point of the actuator's shaft (revolute joint);

(ix) $\mathbf{A}_{\mathbf{i}} \mathbf{C}_{\mathbf{i}}=\left[A_{i} C_{i x}, A_{i} C_{i y}, A_{i} C_{i z}\right]^{T}$ is the vector from point $A_{i}$ to point $C_{i}$ of the $i$ th upper attachment point of its shaft (revolute joint); it should be noted that $\left|\mathbf{A}_{\mathbf{i}} \mathbf{C}_{\mathbf{i}}\right|=l_{A i C i}$ represents various lengths for each linkage;

(x) $\mathbf{A}_{\mathbf{i}} \mathbf{P}=\left[A_{i} P_{x}, A_{i} P_{y}, A_{i} P_{z}\right]^{T}$ is the vector from point $A_{i}$ to the origin of the moving platform $P$; it should be noted that $\left|\mathbf{A}_{\mathbf{i}} \mathbf{P}\right|=l_{\text {AiP }}$ represents various lengths for each linkage;

(xi) $P_{p i}$ and $A_{i}^{\prime}$ are geometrical points of intersection $i$ th support revolute joint axis with, respectively, the symmetry axis of the $i$ th actuators shaft and horizontal plane set at the level $Z_{B i}$, determined by three centre points of the revolute joints $B_{i}$;

(xii) $\mathbf{P}_{\mathbf{p i}} \mathbf{A}_{\mathbf{i}}=\left[P_{P i} A_{i x}, P_{P i} A_{i y}, P_{P i} A_{i z}\right]^{T}$ is the vector from point $P_{P i}$ to $A_{i}$ of the micromanipulator's $i$ th arm;

(xiii) $\mathbf{P}_{\mathbf{p i}} \mathbf{C}_{\mathbf{i}}=\left[P_{P i} C_{i x}, P_{p i} C_{i y}, P_{P i} C_{i z}\right]^{T}$ is the vector from point $P_{P i}$ to $C_{i}$ of the micromanipulator's $i$ th arm;

(xiv) $\mathbf{P}_{\mathbf{b i}}^{\mathbf{n}}=\left[P_{b i x}^{n}, P_{b i y}^{n}, P_{b i z}^{n}\right]$ and $\mathbf{P}_{\mathbf{b i}}^{\tau}=\left[P_{b i x}^{\tau}, P_{b i y}^{\tau}, P_{b i z}^{\tau}\right]$ represent vectors normal and tangent to the $i$ th actuator;

(xv) $\beta_{i}$ defines the $i$ th actuator's tilt relative to the base's horizontal plane, $\theta_{i}$ represents the rotation of the 
moving platform's $i$ th revolute joint, and $\alpha$ and $\varphi$ determine the yaw and pitch of the moving frame.

The length of the micromanipulator's $i$ th arm is labeled as $\mathbf{1}_{\text {AiCi }}$ (drawn in Figure 3). It corresponds to the following lengths of all three struts $l_{A 1 C 1}, l_{A 2 C 2}$, and $l_{A 3 C 3}$. Its measurement starts from point $A_{i}\left(x_{A i}, y_{A i}, z_{A i}\right)$ being the place of attachment of the selected arm to the fixed platform and point $C_{i}\left(x_{C i}, y_{C i}, z_{C i}\right)$ which forms the geometric center of the rotary joint connecting the moving platform unit ring with a selected robot arm. In the presented system the linear extension $\mathbf{l}_{\mathbf{i}}$ of the $i$ th piezoelectric actuators is measured.
Taking into consideration all the geometrical dependencies shown in Figures 3 and 4, as a result, the extension of the $i$ th piezoelectric actuator $\mathbf{l}_{\mathbf{i}}$ has been obtained:

$$
\mathbf{l}_{\mathbf{i}}=\left[\begin{array}{l}
l_{A_{1} B_{1}} \cos \left(\beta_{1}+\frac{\pi}{4}\right)+\sqrt{l_{A_{1} B_{1}}^{2}\left(\cos ^{2}\left(\beta_{1}+\frac{\pi}{4}\right)-1\right)+l_{A_{1} C_{1}}^{2}} \\
l_{A_{2} B_{2}} \cos \left(\beta_{2}+\frac{\pi}{4}\right)+\sqrt{l_{A_{2} B_{2}}^{2}\left(\cos ^{2}\left(\beta_{2}+\frac{\pi}{4}\right)-1\right)+l_{A_{2} C_{2}}^{2}} \\
l_{A_{3} B_{3}} \cos \left(\beta_{3}+\frac{\pi}{4}\right)+\sqrt{l_{A_{3} B_{3}}^{2}\left(\cos ^{2}\left(\beta_{3}+\frac{\pi}{4}\right)-1\right)+l_{A_{3} C_{3}}^{2}}
\end{array}\right],
$$

where

$$
\begin{aligned}
& \mathbf{1}_{\mathrm{AiCi}}=\left[\sqrt{\frac{\sqrt{\left(\sqrt{x^{2}+(y-R)^{2}}-r\right)^{2}+\left(z-z_{A 1}\right)^{2}}}{\left.\sqrt{\left(x-\frac{\sqrt{3}}{2} R\right)^{2}+\left(y+\frac{1}{2} R\right)^{2}}-r\right)^{2}+\left(z-z_{A 2}\right)^{2}}}\right], \\
& \cos \left(\beta_{1}+\frac{\pi}{4}\right)=\frac{1}{\sqrt{2\left(\left(\left(z-z_{B 1}\right) / \sqrt{x^{2}+(y-R)^{2}}\right)^{2}+1\right)}} \\
& -\frac{\left(z-z_{B 1}\right) / \sqrt{x^{2}+(y-R)^{2}}}{\sqrt{2\left(\left(\left(z-z_{B 1}\right) / \sqrt{x^{2}+(y-R)^{2}}\right)^{2}+1\right)}}, \\
& \begin{aligned}
\cos \left(\beta_{2}+\frac{\pi}{4}\right)= & \frac{1}{\sqrt{2\left(\left(\left(z-z_{B 2}\right) / \sqrt{\left.(x-(\sqrt{3} / 2) R)^{2}+(y+(1 / 2) R)^{2}\right)^{2}+1}\right)\right.}} \\
& -\frac{\left(z-z_{B 2}\right) / \sqrt{(x-(\sqrt{3} / 2) R)^{2}+(y+(1 / 2) R)^{2}}}{\left.\sqrt{2\left(\left(z-z_{B 2}\right) / \sqrt{x^{2}+\sqrt{(x-(\sqrt{3} / 2) R)^{2}+(y+(1 / 2) \mathrm{R})^{2}}(y-R)^{2}}\right)^{2}}+1\right)}
\end{aligned} \\
& \cos \left(\beta_{3}+\frac{\pi}{4}\right)=\frac{1}{\sqrt{2\left(\left(\left(z-z_{B 3}\right) / \sqrt{\left.(x+(\sqrt{3} / 2) R)^{2}+(y-(1 / 2) R)^{2}\right)^{2}+1}\right)\right.}} \\
& \left(z-z_{B 3}\right) / \sqrt{(x+(\sqrt{3} / 2) R)^{2}+(y-(1 / 2) R)^{2}} \\
& \sqrt{2\left(\left(\left(z-z_{B 3}\right) / \sqrt{x^{2}+\sqrt{(x+(\sqrt{3} / 2) R)^{2}+(y-(1 / 2) R)^{2}}(y-R)^{2}}\right)^{2}+1\right)}
\end{aligned}
$$




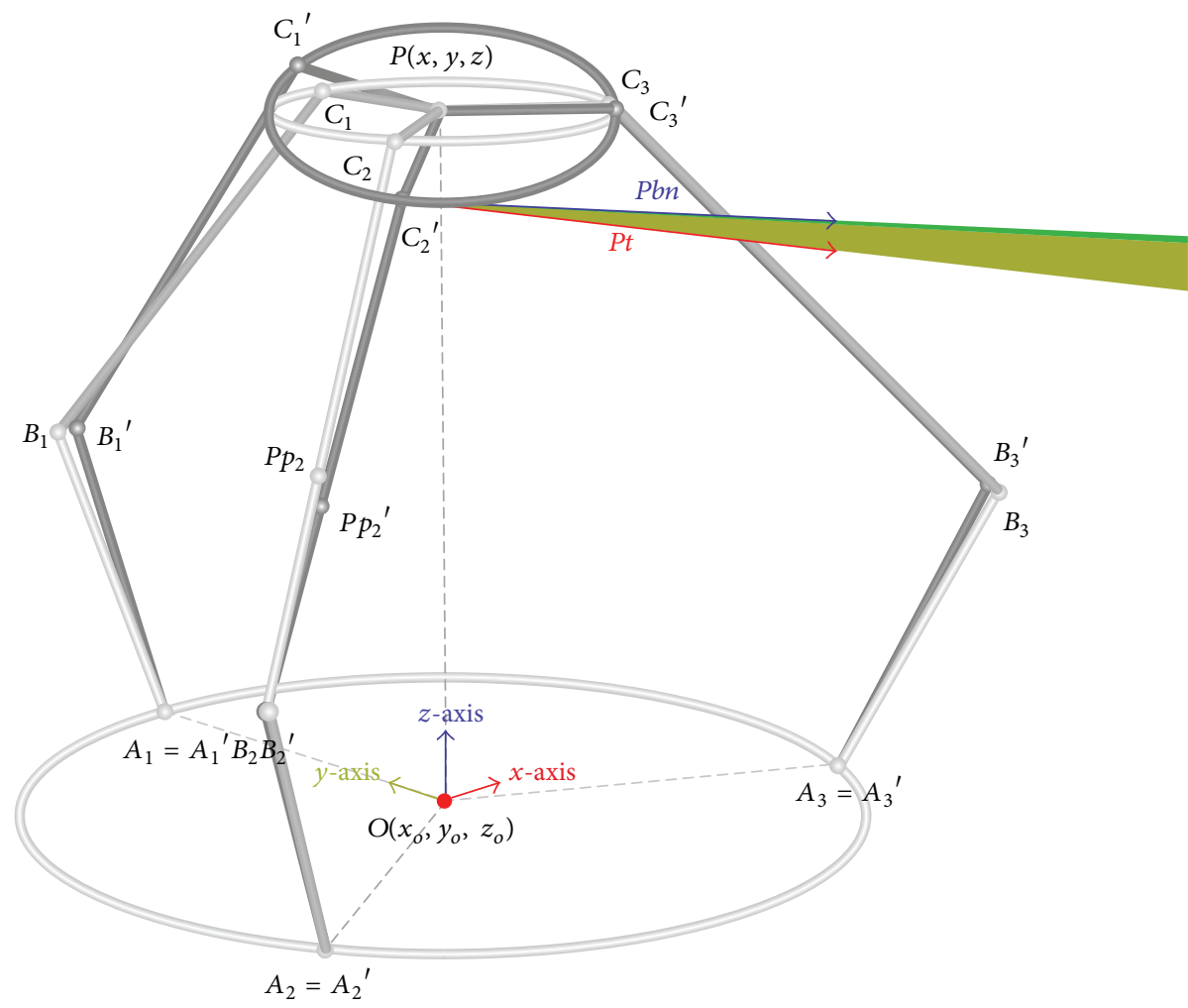

FIGURE 7: Cabri 3D geometric model of micromanipulator with indicated angular translation $\delta$ of piezoelectric drive.

To find the angular $\delta_{i}$, by which the $i$ th piezoelectric actuator has to move to overcome the deviations of the moving platform, the analytic geometry analyses were used. It was necessary to appoint two vectors, $\mathbf{P}_{b}^{n}$ normal to the main axis of symmetry of the actuator's shaft and $\mathbf{P}^{\boldsymbol{\tau}}$ tangent to the platform at the point of joining with the right arm of the micromanipulator, and then determine the angle between them (Figure 5). This angle corresponds to the actual rotational $\delta_{i}$ displacement of the rotor, relative to its initial position, resulting from the perfectly leveled microrobot's moving platform in horizontal position. Given all the geometrical dependencies and the distribution of the linkages, the angular displacement of the piezoelectric actuator's shaft $\delta_{i}$ has been received:

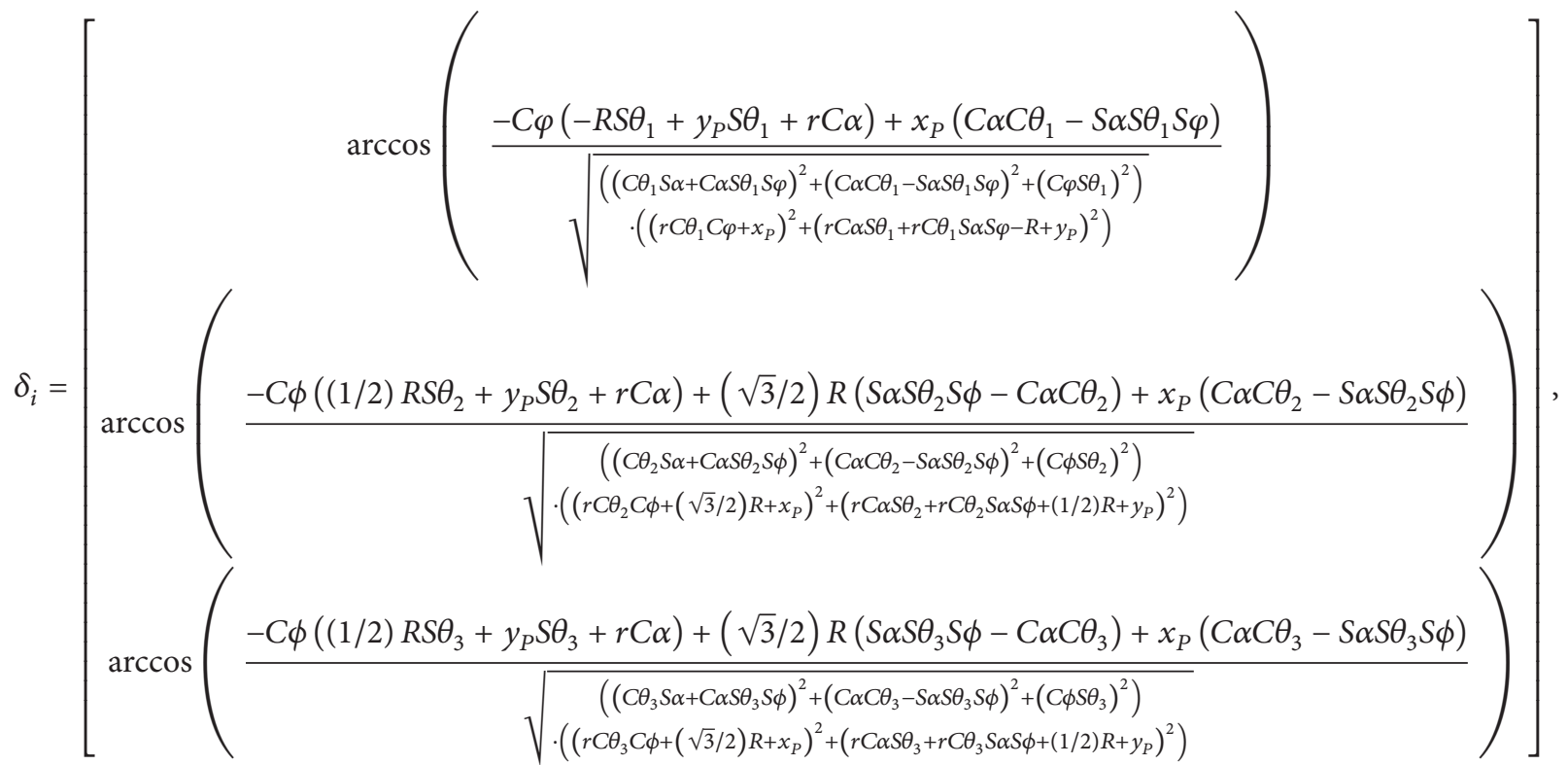


where

$$
\begin{array}{cc}
S \alpha=\sin (\alpha), & C \alpha=\cos (\alpha), \\
S \varphi=\sin (\varphi), & C \varphi=\cos (\varphi), \\
S \theta=\sin (\theta), & C \theta=\cos (\theta), \\
\theta_{1}=\pi+2 \cdot \arctan \left(\frac{A_{1}}{B_{1}}+\sqrt{\frac{A_{1}^{2}+B_{1}^{2}}{B_{1}^{2}}}\right),
\end{array}
$$

$A_{1}$

$$
\begin{aligned}
& =C \varphi \cdot x_{P}+S \varphi \\
& \quad\left(S \alpha \cdot\left(R-y_{P}\right)\right. \\
& \left.\quad+C \alpha \cdot\left(z_{P}-\frac{r \cdot\left(z_{P}-z_{B}\right)}{\sqrt{x_{P}^{2}+\left(y_{P}-R\right)^{2}}}\right)\right), \\
& B_{1}=C \alpha \cdot\left(y_{P}-R\right) \\
& \quad+S \alpha \cdot\left(z_{P}-\frac{r \cdot\left(z_{P}-z_{B}\right)}{\sqrt{x_{P}^{2}+\left(y_{P}-R\right)^{2}}}\right), \\
& \theta_{2}=2 \cdot \arctan \left(\frac{A_{2}}{B_{2}}-\sqrt{\frac{A_{2}^{2}+B_{2}^{2}}{B_{2}^{2}}}\right),
\end{aligned}
$$

$A_{2}$

$$
\begin{aligned}
= & -C \varphi \cdot\left(\frac{\sqrt{3}}{2} R+x_{P}\right)+S \varphi \\
& \cdot\left(-S \alpha \cdot\left(\frac{1}{2} R+y_{P}\right)+C \alpha\right. \\
& \left.\left(z_{P}-\frac{r \cdot\left(z_{P}-z_{B}\right)}{\sqrt{\left(x_{P}+(\sqrt{3} / 2) R\right)^{2}+\left(y_{P}+(1 / 2) R\right)^{2}}}\right)\right),
\end{aligned}
$$

$B_{2}$

$$
\begin{gathered}
=C \alpha \cdot\left(\frac{1}{2} R-y_{P P}\right)+S \alpha \\
\cdot\left(z_{P}-\frac{r \cdot\left(z_{P}-z_{B}\right)}{\sqrt{\left(x_{P}+(\sqrt{3} / 2) R\right)^{2}+\left(y_{P}+(1 / 2) R\right)^{2}}}\right), \\
\theta_{3}=2 \cdot \arctan \left(\frac{A_{3}}{B_{3}}-\sqrt{\frac{A_{3}^{2}+B_{3}^{2}}{B_{3}^{2}}}\right),
\end{gathered}
$$
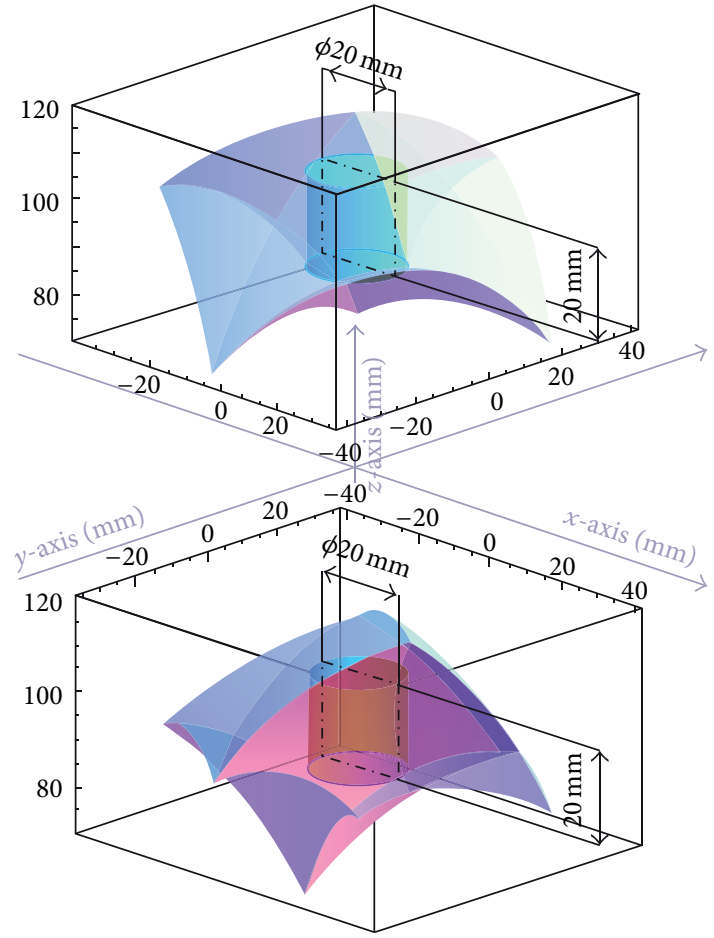

FIGURE 8: Shape and size of the microrobot's workspace.

$A_{3}$

$$
=C \varphi \cdot\left(\frac{\sqrt{3}}{2} R-x_{P}\right)+S \varphi
$$$$
\left(-S \alpha \cdot\left(\frac{1}{2} R+y_{P}\right)+C \alpha\right.
$$

$$
\left.\cdot\left(z_{P}-\frac{r \cdot\left(z_{P}-z_{B}\right)}{\sqrt{\left(x_{P}+(\sqrt{3} / 2) R\right)^{2}+\left(y_{P}+(1 / 2) R\right)^{2}}}\right)\right),
$$

$$
\begin{aligned}
B_{3}= & C \alpha \cdot\left(\frac{1}{2} R-y_{P P}\right)+S \alpha \\
& \cdot\left(z_{P}-\frac{r \cdot\left(z_{P}-z_{B}\right)}{\sqrt{\left(x_{P}+(\sqrt{3} / 2) R\right)^{2}+\left(y_{P}+(1 / 2) R\right)^{2}}}\right) .
\end{aligned}
$$

\section{Theoretical and Useful Workspace}

The theoretical workspace of the micromanipulator has been determined using Wolfram Mathematica computational software program. The volume has been estimated at $60346\left[\mathrm{~mm}^{3}\right]$, with an error of $39\left[\mathrm{~mm}^{3}\right]$, representing approximately $0,6 \%$ of the specified result. Figure 8 presents the resulting workspace shown as part of all joined together 


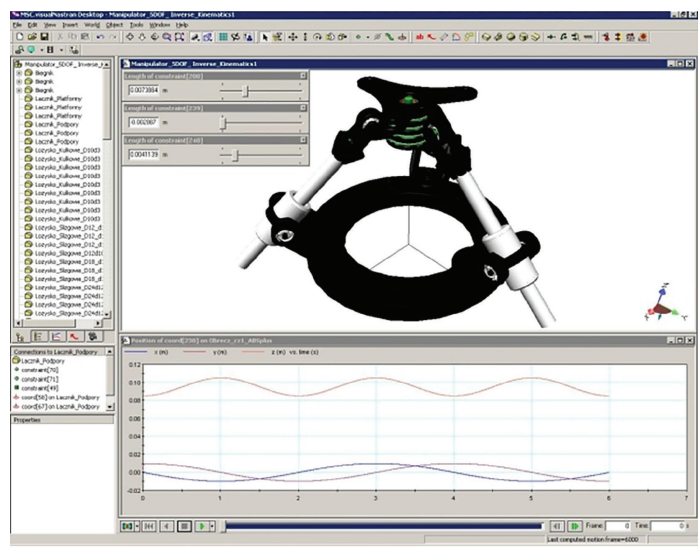

FIgURE 9: Rigid-body model build in Visual Nastran 4D.

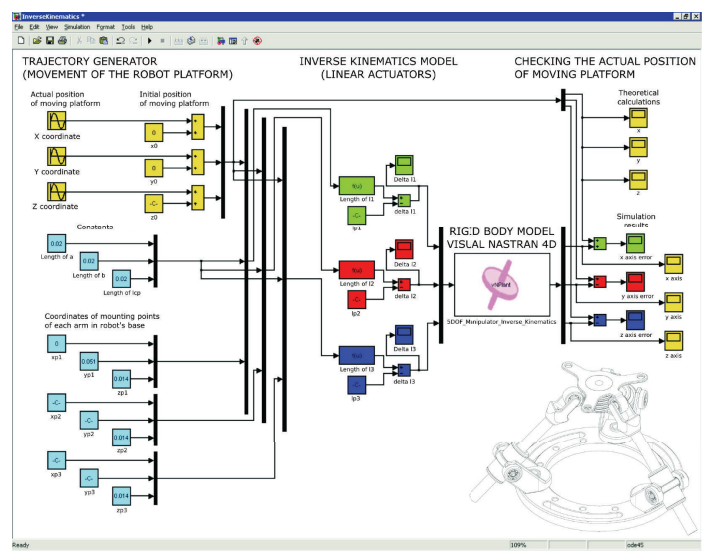

FIgure 10: Computational model developed in MATLAB/Simulink.

possible positions, that can be achieved by the centre of the moving platform. Established useful workspace, achievable by the micromanipulator, has the form of a cylinder with a radius of $R=10[\mathrm{~mm}]$, height $H=25[\mathrm{~mm}]$, and volume equal to $6280\left[\mathrm{~mm}^{3}\right]$.

\section{Verification of the Mathematical Model}

In order to verify the obtained mathematical relations between the position of the moving platform and the extension of the piezoelectric drives, a virtual prototype of the manipulator has been used. For this purpose, a rigid-body model of the mechanism was built in Visual Nastran 4D and a computational model developed in MATLAB/Simulink. Both models are presented in Figures 9 and 10, respectively. The aim of the simulation was to determine the expected maximum stroke (extension) of each used actuator and specify the correctness of the mathematical model of the manipulator's inverse kinematics.

A test of the correctness of the calculations model was performed by making the moving platform of the virtual 3D model follow two different trajectories, obtained by combining three sine waves in all three directions of a Cartesian system, but with different frequencies. Mathematical

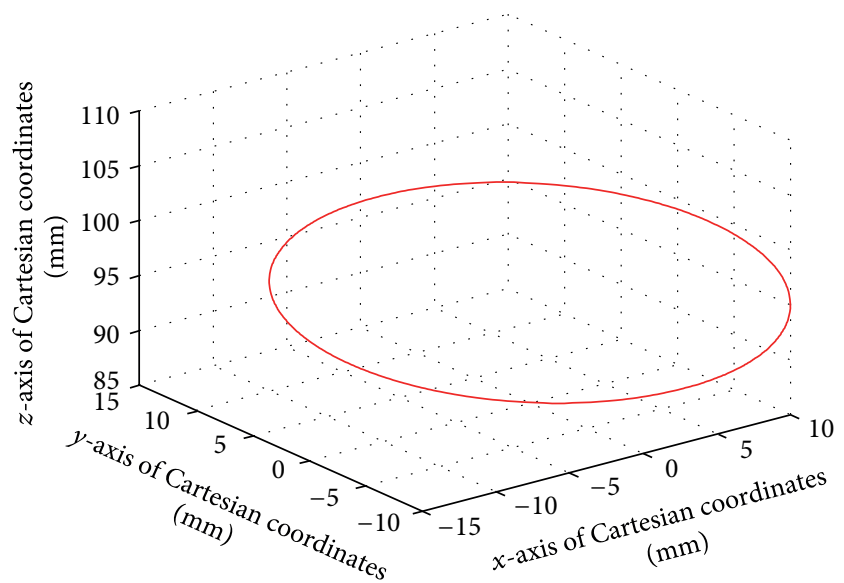

Figure 11: Theoretical movement path of the moving platform.

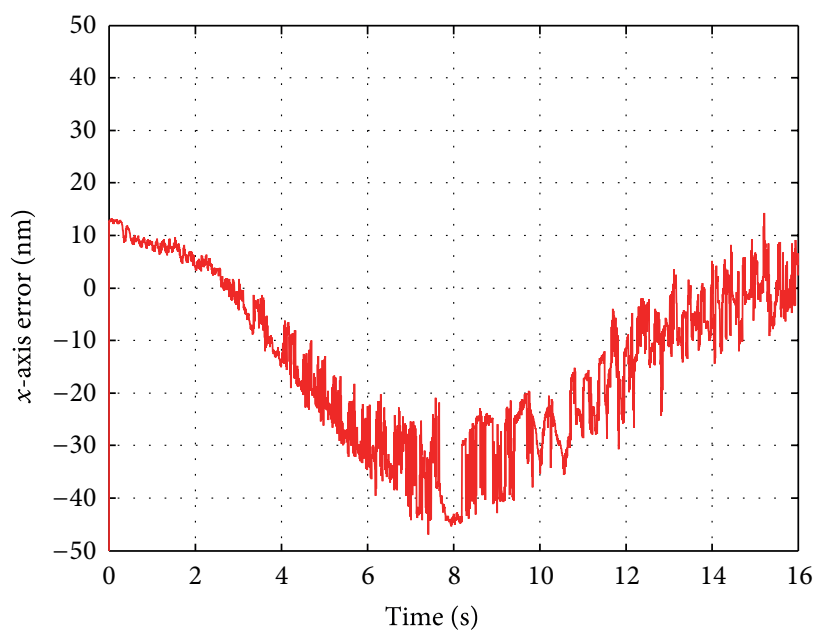

FIGURE 12: Error generated by computational model in $X$-axis.

model calculations were performed for different levels of discretization. Graphical representation of the selected path of the microrobot's head is shown in Figure 11.

Figures 12, 13, and 14 present volumes of received errors. Both types of computational software, used as simulation environments, arbitrarily set the point of precision in performed calculations, which may differ. The lesser the step of discretization is, the more accurate the result can be obtained. Thus errors arise directly from the approximations and rounding used by cosimulation of MATLAB/Simulink with Visual Nastran 4D. In addition, assembly tolerances and the bond between the elements of the rigid-body model must be taken into consideration.

In order to verify the mathematical model of the manipulator, describing angular displacement of 2DOF piezoelectric actuators, symbolic-numerical software Wolfram Mathematica and solid geometry software Cabri Geometry 3D was used. Correctness of the obtained final relations, describing the extension and rotation of the piezoelectric actuator, in symbolic and then numerical form, was confirmed by Wolfram Mathematica computational program, as shown in Figure 15. Cabri Geometry 3D software was used to create a 
TABLE 1: Example results of the inverse kinematics for rotational movement of the piezoelectric actuators.

\begin{tabular}{|c|c|c|c|c|c|c|c|c|c|c|c|c|}
\hline & \multicolumn{12}{|c|}{ Angles } \\
\hline & $\delta_{1}\left[{ }^{\circ}\right]$ & $\delta_{2}\left[{ }^{\circ}\right]$ & $\delta_{3}\left[{ }^{\circ}\right]$ & $\delta_{1}\left[{ }^{\circ}\right]$ & $\delta_{2}\left[{ }^{\circ}\right]$ & $\delta_{3}\left[{ }^{\circ}\right]$ & $\delta_{1}\left[{ }^{\circ}\right]$ & $\delta_{2}\left[{ }^{\circ}\right]$ & $\delta_{3}\left[{ }^{\circ}\right]$ & $\delta_{1}\left[{ }^{\circ}\right]$ & $\delta_{2}\left[{ }^{\circ}\right]$ & $\delta_{3}\left[{ }^{\circ}\right]$ \\
\hline Mathematica & 17.10 & 25.43 & 10.59 & 12.15 & 17.85 & 6.39 & 6.53 & 9.33 & 2.88 & 1.39 & 1.92 & 0.53 \\
\hline Cabri 3D & 16.89 & 25.73 & 10.51 & 12.14 & 17.98 & 6.26 & 6.56 & 9.35 & 2.82 & 1.40 & 1.92 & 0.52 \\
\hline Error & 0.21 & -0.30 & 0.08 & 0.01 & -0.13 & 0.13 & -0.03 & -0.02 & 0.06 & -0.01 & 0.00 & 0.01 \\
\hline$\alpha$ & & $-15^{\circ}$ & & & $-10^{\circ}$ & & & $-5^{\circ}$ & & & $-1^{\circ}$ & \\
\hline$\beta$ & & $-15^{\circ}$ & & & $-10^{\circ}$ & & & $-5^{\circ}$ & & & $-1^{\circ}$ & \\
\hline Mathematica & 26.91 & 5.41 & 30.22 & 16.65 & 4.10 & 20.07 & 7.68 & 2.31 & 9.90 & 1.44 & 0.51 & 1.94 \\
\hline Cabri 3D & 25.85 & 5.82 & 29.94 & 16.23 & 4.26 & 19.94 & 7.56 & 2.42 & 9.88 & 1.44 & 0.51 & 1.94 \\
\hline Error & 1.06 & -0.41 & 0.28 & 0.42 & -0.16 & 0.13 & 0.12 & -0.11 & 0.02 & 0.00 & 0.00 & 0.00 \\
\hline$\alpha$ & & $15^{\circ}$ & & & $10^{\circ}$ & & & $5^{\circ}$ & & & $1^{\circ}$ & \\
\hline$\beta$ & & $-15^{\circ}$ & & & $-10^{\circ}$ & & & $-5^{\circ}$ & & & $-1^{\circ}$ & \\
\hline Mathematica & 16.88 & 10.59 & 25.43 & 12.15 & 6.37 & 17.85 & 6.53 & 2.88 & 9.33 & 1.39 & 0.53 & 1.92 \\
\hline Cabri 3D & 17.10 & 10.56 & 25.81 & 12.17 & 6.39 & 18.01 & 6.58 & 2.87 & 9.39 & 1.40 & 0.53 & 1.93 \\
\hline Error & -0.22 & 0.03 & -0.38 & -0.02 & -0.02 & -0.16 & -0.05 & 0.01 & 0.06 & -0.01 & 0.00 & -0.01 \\
\hline$\alpha$ & & $-15^{\circ}$ & & & $-10^{\circ}$ & & & $-5^{\circ}$ & & & $-1^{\circ}$ & \\
\hline$\beta$ & & $15^{\circ}$ & & & $10^{\circ}$ & & & $5^{\circ}$ & & & $1^{\circ}$ & \\
\hline Mathematica & 26.91 & 30.22 & 5.41 & 16.65 & 20.07 & 4.10 & 7.68 & 9.90 & 2.31 & 1.43 & 1.94 & 0.50 \\
\hline Cabri 3D & 25.85 & 29.97 & 5.76 & 16.24 & 19.97 & 4.21 & 7.59 & 9.91 & 2.32 & 1.44 & 1.94 & 0.51 \\
\hline Error & 1.06 & 0.25 & -0.35 & 0.41 & 0.10 & -0.11 & 0.09 & -0.01 & -0.01 & -0.01 & 0.00 & -0.01 \\
\hline$\alpha$ & & $15^{\circ}$ & & & $10^{\circ}$ & & & $5^{\circ}$ & & & $1^{\circ}$ & \\
\hline$\beta$ & & $15^{\circ}$ & & & $10^{\circ}$ & & & $5^{\circ}$ & & & $1^{\circ}$ & \\
\hline
\end{tabular}

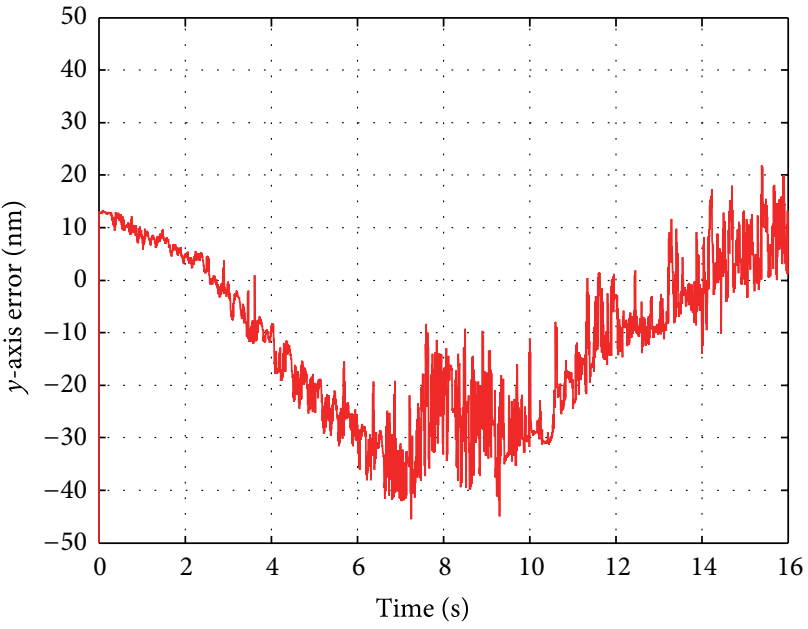

FIGURE 13: Error generated by computational model in $Y$-axis.

simplified geometric model of the micromanipulator in order to study changes in angular displacement of the individual piezoelectric drives of the microrobot, resulting from a change in position and orientation of the moving platform movement, as shown in Figure 16. The reference solutions of the inverse kinematic were obtained from Wolfram Mathematica.

The correctness of the results, gained by using this computational environment, has been confirmed by the analysis of the boundary points, for which error value tends to be zero.

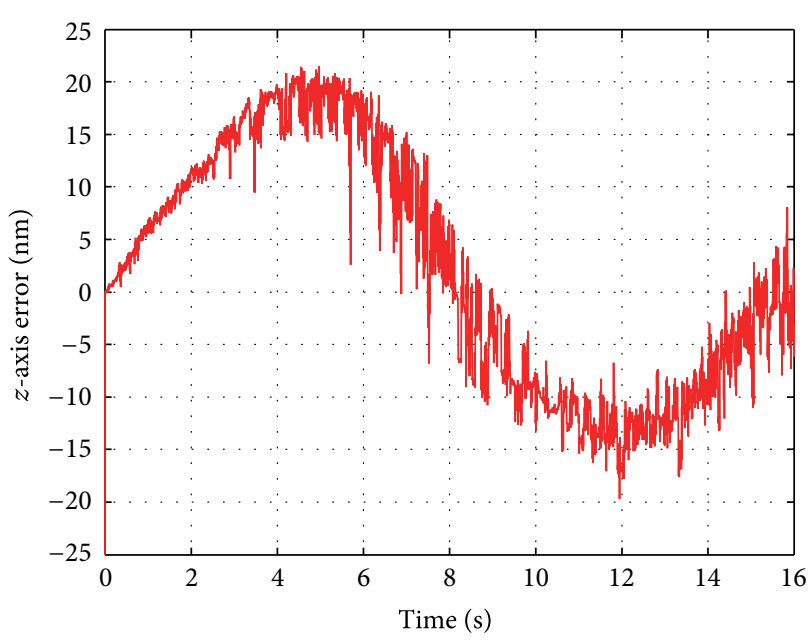

FIGURE 14: Error generated by computational model in $Z$-axis.

The obtained results are shown in Table 1, which presents the errors resulting from the approximations used by Cabri Geometry 3D.

The resulting angular displacement of $\delta_{1}, \delta_{2}$, and $\delta_{3}$ is characterized by an average error weight at $0,16 \%$. The maximal error values, recorded for the maximal angular tilts of the moving platform in $X$ - and $Y$-axis $-\alpha=15^{\circ}$ and $\beta=15^{\circ}$, are equal to $7,04 \%$, while when $\alpha=1^{\circ}$ and $\beta=1^{\circ}$, the error is negligibly low. 


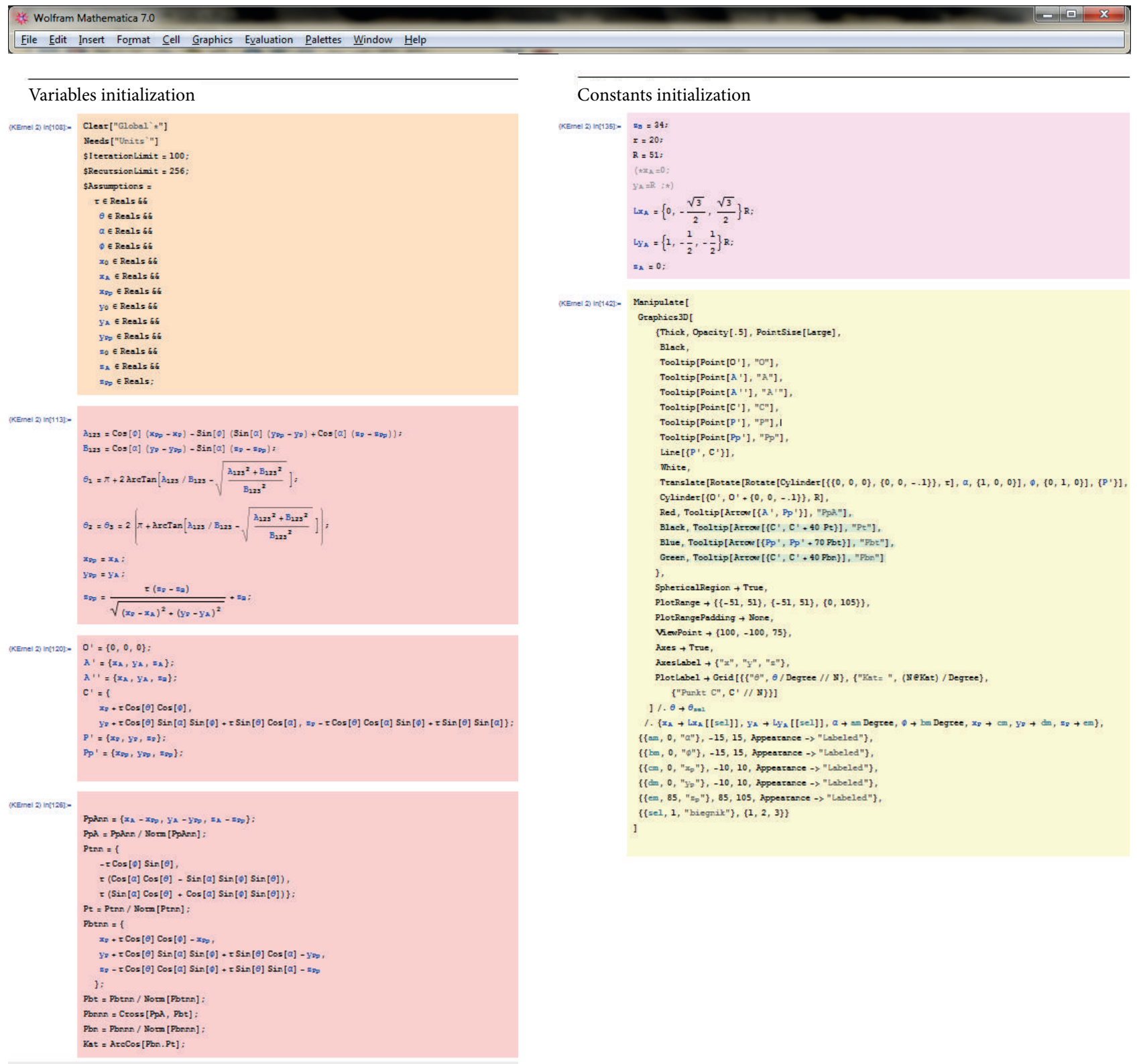

FIGURE 15: Mathematical model of the inverse kinematics implemented in Wolfram Mathematica computational program.

\section{Conclusions}

The objective of this work was to introduce a structural and geometrical model of a novel 5-degree-of-freedom spatial parallel micromanipulator. Analytical solutions of the microrobot's inverse kinematics tasks as well as analysis of the effective and useful workspace of the micromechanism were presented. Finally verification of the geometric model correctness using a multibody analysis program Visual Nastran 4D, interactive geometry software Cabri Geometry 3D, computation environment MATLAB/Simulink, and a computational software program Wolfram Mathematica was made. Mathematical model of microrobot's structure was provided by using analytic geometry problems, infinitesimal calculus, and vector calculus.
The greatest achievement presented in this study was to determine a detailed solution of the inverse kinematics task for the micromanipulator in an analytical form, which can be used for control and trajectory generator. The received mathematical model describes the behaviour of individual elements for the applied 2-DOF piezoelectric actuator, resulting from the translation and orientation of the microrobot's platform. The model is highly complex and contains a number of elements generating errors either on the computational engine side (numerical errors resulting from approximations) or possibly used simplifications for easier implementation for the control system and trajectory generator. It is possible to increase the accuracy of the model by performing equations with feedback from the obtained results, thus establishing the error calculations and 


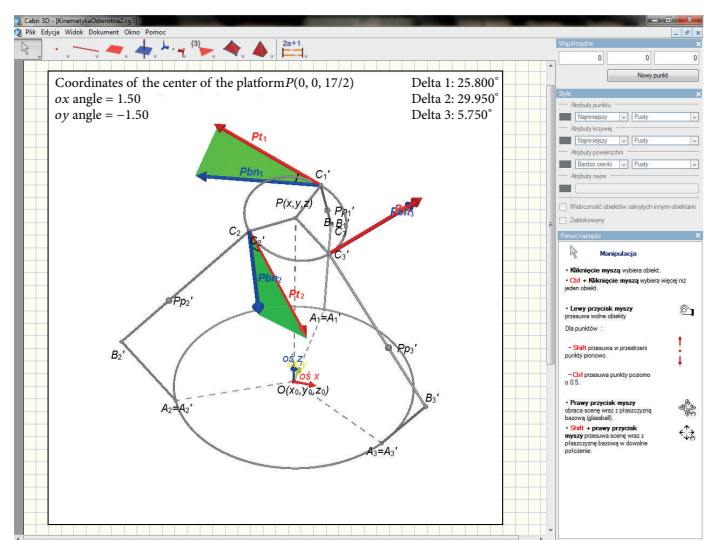

Figure 16: Geometric model build in Cabri Geometry 3D.

the iterative reduction to a satisfactory level [18]. Nevertheless, the verification methods of the obtained computational model showed that the analytical equations have been derived correctly. Errors generated during the virtual prototype test result from the capabilities and accuracy of computing environments and also form simplifications in the models used by the programs for numerical computation. It should be noted that (in the authors' opinion) the received errors are minor and acceptable for the project.

\section{Conflict of Interests}

The authors have declared that no conflict of interests exists.

\section{References}

[1] J. P. Merlet, Solid Mechanics and It's Applications: Parallel Robots, Kluwer Academic, Springer, 2000.

[2] D. Prusak, T. Uhl, and M. Petko, Introduction to the Problem of Microrobotics, vol. 5, Measurements, Automation, Control PAK, Polish Society of Automation Measurements and Robotics POLSPAR, 2004.

[3] G. Gogu, Structural Synthesis of Parallel Robots, Part 1, Springer, 2008.

[4] D. Prusak and T. Uhl, "Design and control of a novel type hybrid micromanipulator with piezoelectric actuators and vision feedback system," in Proceedings of the 12th Congress of theoretical and Applied Mechanics, Adelaide, Australia, August 2008.

[5] D. Prusak, T. Uhl, and M. Petko, "Parallel microrobotsconcept and prototyping," in Proceedings of the 12th IEEE international conference on Methods and Models in Automation and Robotics, Technical University of Szczecin, Institute of Control Engineering, IEEE, IEEE Robotics \& Automation Society, IEEE Control System Society, Miedzyzdroje, Poland, August 2006.

[6] G. Karpiel and M. Petko, Three-armed parallel manipulator, AGH University of Science and Technology, Krakow, Poland, Polish patent no. PL, 208563 B1, 31. 05. 2011, Int. Cl.: B25J 18/04 (2006. 01).

[7] G. Karpiel and M. Petko, Triple actuated joint, AGH University of Science and Technology, Krakow, Poland, Polish patent no. PL, 207396 B1, 31. 12. 2010, Int. Cl. F16C 11/00 (2006. 01).
[8] G. Karpiel, M. Petko, and T. Uhl, Three-arm parallel manipulator, AGH University of Science and Technology, Krakow, Poland, Polish patent no. PL, 203631 B1, 30. 10. 2009, Int. Cl.: B25J 18/00 (2006. 01).

[9] D. Prusak, M. Petko, and G. Karpiel, Three-armed parallel manipulator, AGH University of Science and Technology, Krakow, Poland, Polish patent no. PL, 210002 B1, 30. 11. 2011, Int. Cl.: B25J 18/04 (2006. 01).

[10] Y. Yun and Y. Li, "Design and analysis of a novel 6-DOF redundant actuated parallel robot with compliant hinges for high precision positioning," Nonlinear Dynamics, vol. 61, no. 4, pp. 829-845, 2010.

[11] J. Huang and Y. Li, "Design and analysis of a completely decoupled compliant parallel XY micro-motion stage," in Proceedings of the IEEE International Conference on Robotics and Biomimetics (ROBIO '10), pp. 1008-1013, Tianjin, China, December 2010.

[12] M. Petko and G. Karpiel, "Mechatronic design of parallel manipulators," in Proceedings of the 3rd IFAC Symposium on Mechatronic Systems, pp. 433-438, Sydney, Australia, September 2004.

[13] M. Petko, G. Karpiel, D. Prusak, and A. Martowicz, "A new 3DOF parallel manipulator," in Proceedings of the 4th International Workshop on Robot Motion and Control (RoMoCo '04), pp. 127-132, June 2004.

[14] J. A. Carretero, R. P. Podhorodeski, M. A. Nahon, and C. M. Gosselin, "Kinematic analysis and optimization of a new three degree-of-freedom spatial parallel manipulator," Journal of Mechanical Design, Transactions of the ASME, vol. 122, no. 1, pp. 17-24, 2000.

[15] D. Jakobović and L. Budin, "Forvard Kinematics of a Steward Platform Mechanism," Problem-Solving Environments in Engineering.

[16] M. Petko, G. Karpiel, D. Prusak, and A. Martowicz, Kinematics of the New Type 3-DOF Parallel Manipulator, vol. 5, Measurements, Automation, Control PAK, Polish Society of Automation Measurements and Robotics POLSPAR, 2004.

[17] D. Prusak, M. Petko, G. Karpiel, and A. Martowicz, "Calibration of a tripod parallel robot," in Advancement in Robotics, Control of Robots with Perception of the Environment, Collective Work, K. Tchoń, Ed., pp. 323-332, Transport and Communication Printing House LLC, Warsaw, Poland, 2005.

[18] K. Gac, G. Karpiel, and M. Petko, "FPGA based hardware accelerator for calculations of the parallel robot inverse kinematics," in Proceedings of the 17th IEEE International conference on Emerging Technologies \& Factory Automation, Krakow, Poland, September2012.

[19] Z. M. Bi, "Development and control of a 5-axis reconfigurable machine tool," Journal of Robotics, vol. 2011, Article ID 583072, 9 pages, 2011.

[20] P. Dario, R. Valleggi, M. C. Carrozza, M. C. Montesi, and M. Cocco, "Microactuators for microrobots: a critical survey," Journal of Micromechanics and Microengineering, vol. 2, no. 3, pp. 141-157, 1992.

[21] Y. Li, J. Huang, and H. Tang, "A compliant parallel xy micromotion stage with complete kinematic decoupling," IEEE Transactions on Automation Science and Engineering, vol. 9, no. 3, 2012.

[22] T. Uhl, M. Mańka, and D. Prusak, "Mechanisms for microand nano-systems," Mechanics, AGH University of Science and Technology, vol. 27, no. 2, pp. 76-83, 2008. 
[23] T. Uhl and D. Prusak, "Microrobots-construction and testing," in Proceedings of the 1st National Conference on Nanoand Micromechanics, Engineering Committee of the Polish Academy of Science PAN, Rzeszow University of Technology, Institute of Fundamental Technological Research of Polish Academy of Science, Krasiczyn, Poland, July 2008.

[24] Alio Industries, "True Nano Precision Motion Systems, Alio Industries," 2012, http://www.alioindustries.com/.

[25] G. B. Chung, J. H. Chung, D. G. Choi, B.-J. Yi, S. Y. Han, and S. J. Kim, "Development of a 5-DOF hybrid micro-manipulator and implementation to needle manipulation process in medical applications," Key Engineering Materials, vol. 326-328, pp. 773776, 2006.

[26] C. Clévy, A. Hubert, J. Agnus, and N. Chaillet, "A micromanipulation cell including a tool changer," Journal of Micromechanics and Microengineering, vol. 15, no. 10, pp. S292-S301, 2005.

[27] CSEM, "Centre Suisse d'Electronique et Microtechnique SA," 2012, http://www.csem.ch/.

[28] D. Chao, G. Zong, and R. Liu, "Design of a 6-DOF compliant manipulator based on serial-parallel architecture," in Proceedings of the IEEE/ASME International Conference on Advanced Intelligent Mechatronics (AIM '05), pp. 765-770, Monterey, Calif, USA, July 2005.

[29] N. R. Fazenda Carriço, Calibration of High-Precision Flexure Parallel Robots, EPFL, Lausanne, Switzerland, 2007.

[30] J. Hesselbach, A. Raatz, J. Wrege, and S. Soetebier, Design and Analyses of a Macro Parallel Robot with Flexure Hinges for Micro Assembly Tasks, Mechatronics \& Robotics, (MechRob), Aachen, Germany, 2004.

[31] J. Hesselbach, J. Wrege, A. Raatz, and O. Becker, "Aspects on design of high precision parallel robots," Assembly Automation, vol. 24, no. 1, pp. 49-57, 2004.

[32] D. Prusak and T. Uhl, "Novel type of hybrid 3-DOF micromanipulator with piezoelectric actuators-mechanical construction and simulations," in Proceedings of the 4th International Conference on Mechatronic Systems and Materials (MSM '08), Bialystok Technical University, Faculty of Mechanical Engineering, Department of Automatics and Robotics, Bialystok, Poland, July 2008.

[33] C. Quaglia, E. Buselli, R. J. Webster III, P. Valdastri, A. Menciassi, and P. Dario, "An endoscopic capsule robot: a mesoscale engineering case study," Journal of Micromechanics and Microengineering, vol. 19, no. 10, Article ID 105007, pp. 1-11, 2009.

[34] D. Prusak and G. Karpiel, "Prototype of micromanipulator with 5DOF," in Proceedings of the 2nd National Conference on nano and micro robotics, Krasiczyn, Rzeszow University of Technology Printing House, Rzeszow, Poland, July 2010.

[35] D. Prusak, G. Karpiel, and T. Uhl, "A novel type of 5 DOF parallel micromanipulator with piezoelectric actuators," in Proceedings of the 13th World Congress in Mechanism and Machine Science, Guanajuato, Mexico, June 2011.

[36] G. Karpiel and D. Prusak, Parallel Robot with 5 Degrees of Freedom, Mechatronic Design: Selected Issues, Collective Work, Edited by Uhl T, AGH University of Science and Technology, Department of Robotics and Mechatronics, Krakow, Poland, 2011.

[37] Z. Kosiński and D. Prusak, Piezomechatronic Actuator Based on Rotational-Linear Converter, Mechatronic Design: Selected Issues, Collective Work, Edited by Uhl T, AGH University of Science and Technology, Department of Robotics and Mechatronics, Krakow, Poland, 2010.
[38] D. Prusak and G. Karpiel, "Prototype of piezoelectric microactuator with two degrees-of-freedom," in Proceedings of the 2nd National Conference on nano and micro robotics, Krasiczyn, Rzeszow University of Technology Printing House, Rzeszow, Poland, July 2010. 

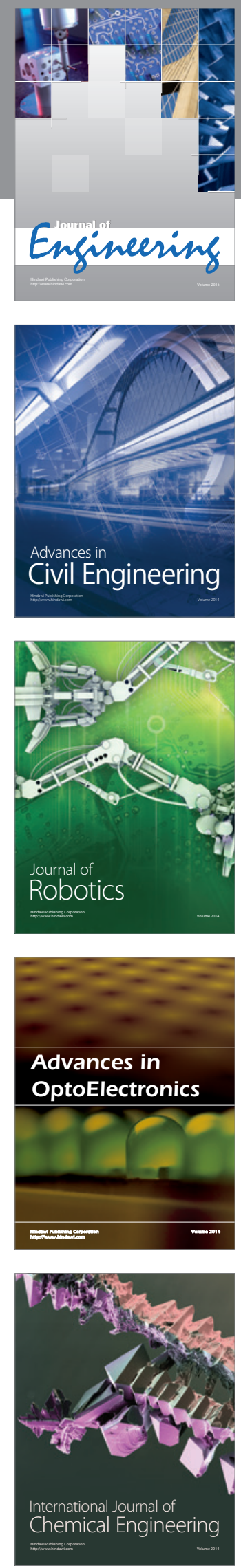

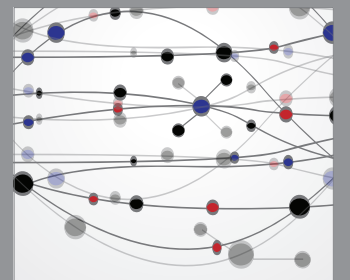

The Scientific World Journal
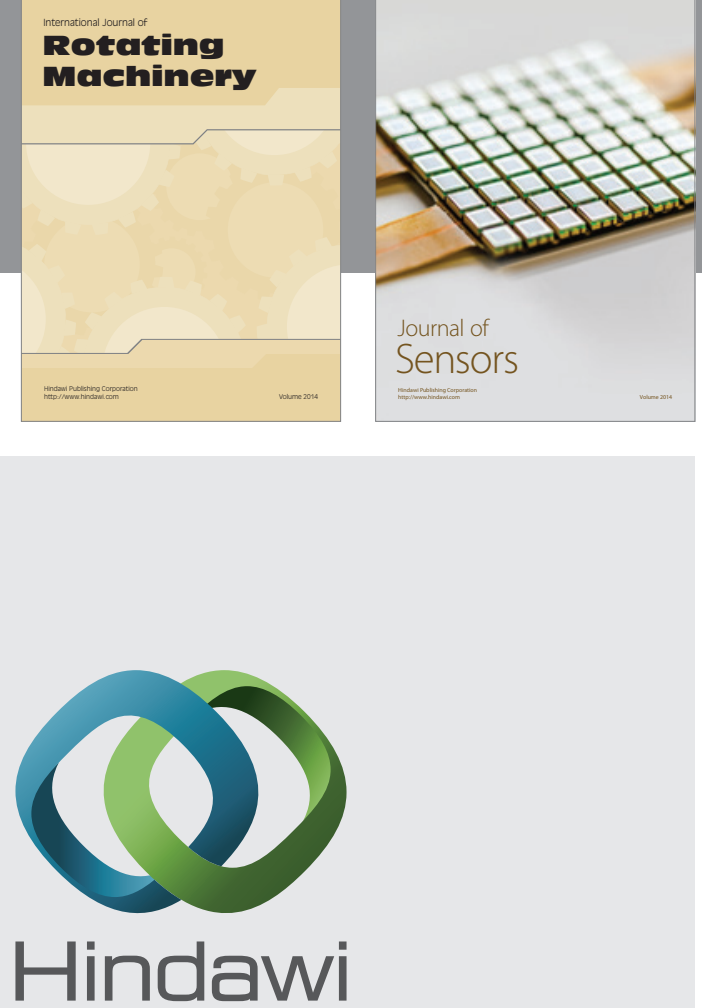

Submit your manuscripts at http://www.hindawi.com
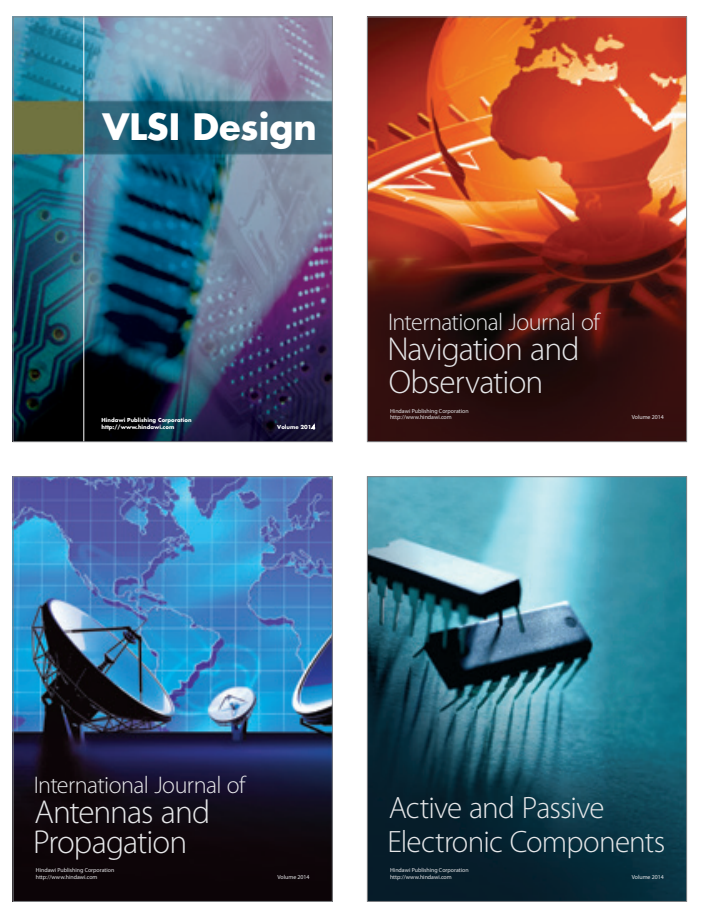
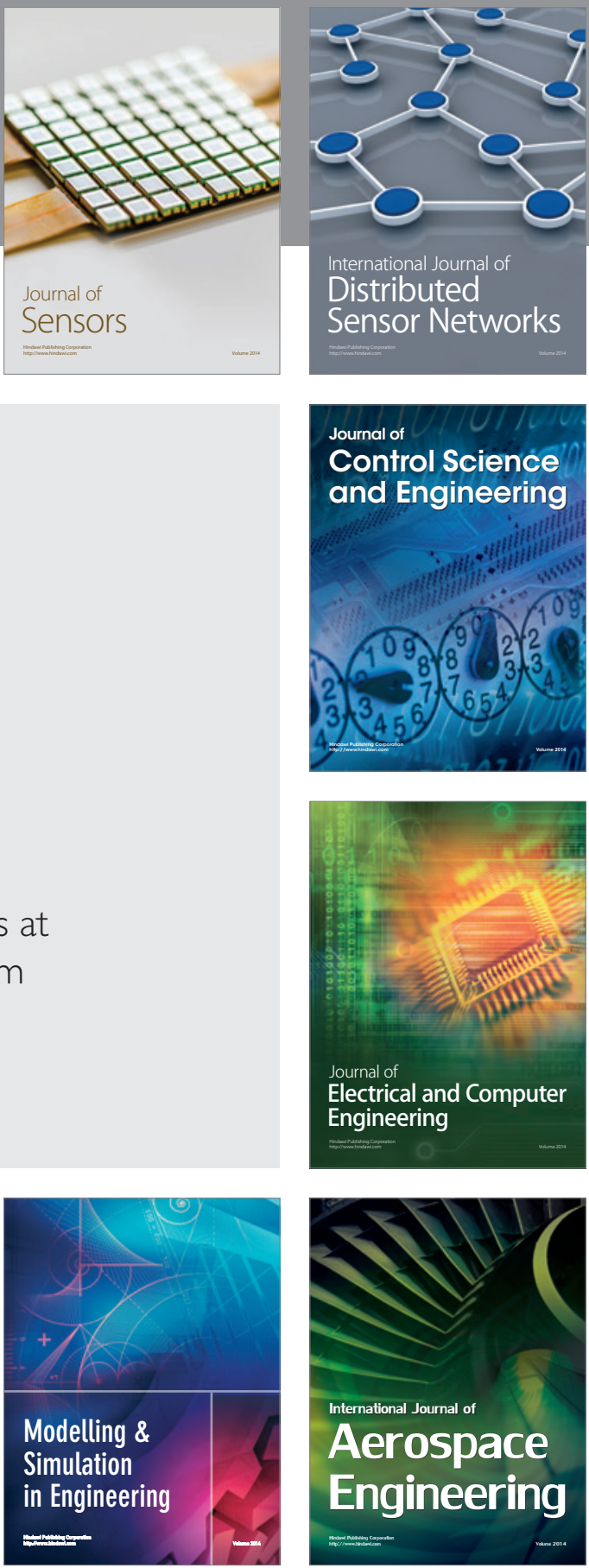

Journal of

Control Science

and Engineering
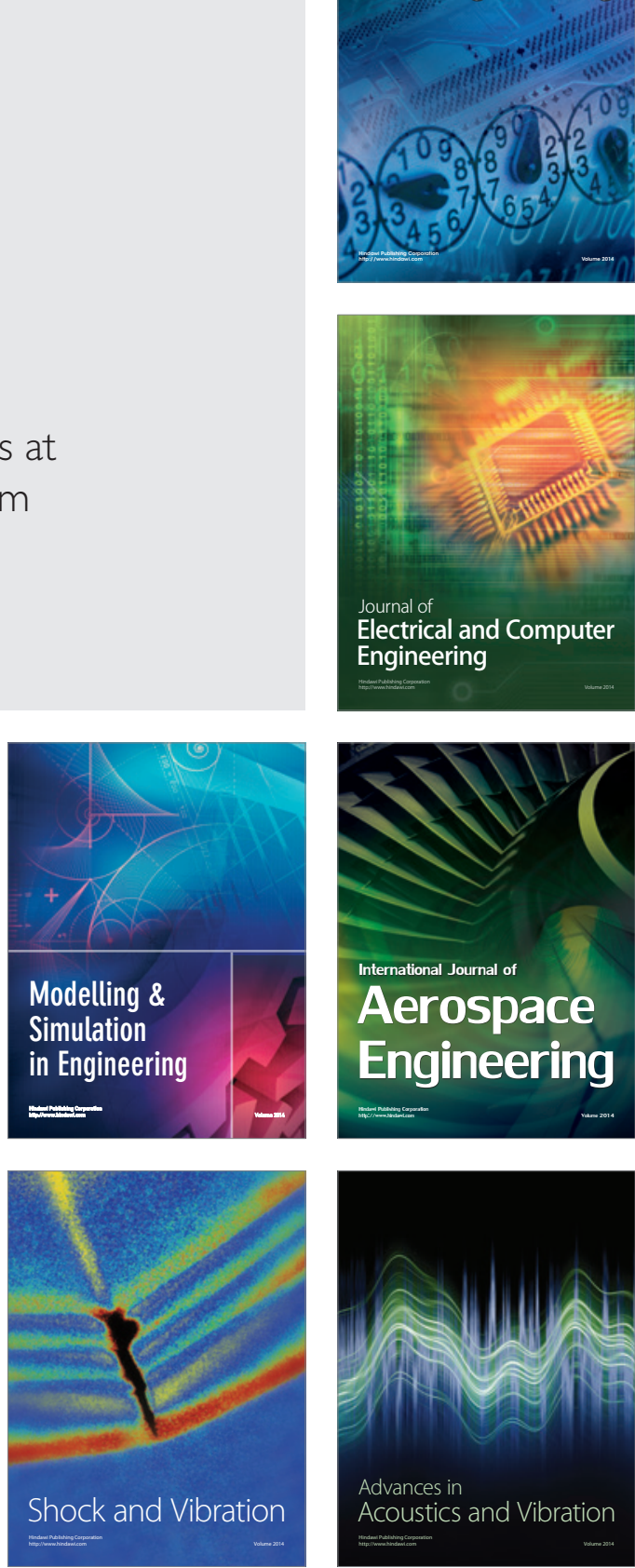